\title{
Communications
}

\section{Regioselective Synthesis of 3-Amino-4-arylisoxazol-5(4H)-ones}

\author{
Dongwon Shin, Ihl Young Choi Lee, and Hee-Jong Lim* \\ Bio-Organic Science Division, Korea Research Institute of Chemical Technology, P.O. Box 107, Yuseong, \\ Daejeon 305-600, Korea. *E-mail: heejong@krict.re.kr \\ Received July 26, 2010, Accepted September 14, 2010
}

Key Words: 3-Amino-4-arylisoxazole-5-(4H)-ones, Thiocarbamoyl ester, Regioselective cyclization

\begin{abstract}
3-Aminoisoxazol-5(4H)-one is an important building block of many biologically active compounds including antimicrobial and antioxidant, ${ }^{1} \mathrm{~K}$ channel opener, ${ }^{2}$ and kinase inhibitors. ${ }^{3} \mathrm{Be}-$ sides, it could be transformed to fused heterocyclic compounds such as indoles, imidazopyridines, ${ }^{4}$ and isoxazolopyrimidines. ${ }^{5}$ Condensation reaction of $\alpha$-cyanoacetate with hydroxylamine was a well known method to access either 3-aminoisoxazol$5(4 H)$-one or isomeric 5-aminoisoxazol-3(4H)-one depending upon condition. ${ }^{6}$ For instance, reaction under the neutral condition provided 3-aminoisoxazolone isomer via acetamidoxime intermediate, whereas 5-aminoisoxazolone isomer was obtained under the alkaline condition. There were only few methods reported in the literature for the preparation of 3-alkyl/arylaminoisoxazoles-5(4H)-ones 3 , such as cyclization of 3-chloroacryloyl chloride, ${ }^{7} \alpha$-amidoximino etser, ${ }^{8}$ and thiocarbamoyl malonate with hydroxylamine. ${ }^{4 a}$ Alkylation of readily available 3-aminoisoxazolone occurred predominantly at isoxazolone ring nitrogen. ${ }^{6 \mathrm{a}}$ Therefore, we were interested in development of new synthetic method to 3-alkyl/arylaminoisoxazol-5(4H)ones 3. We expected the condensation of thiocarbamoyl ester 2 with hydroxylamine in the presence of $\mathrm{Hg}$ (II) or $\mathrm{Ag}$ (I) complex would provide 3 -aminoisoxazolones 3 selectively (Scheme 1). $\mathrm{Hg}(\mathrm{II})$ or $\mathrm{Ag}(\mathrm{I})$ complex have been used for the preparation of amidines and amidoxime via activation of thiocarbonyl. ${ }^{9}$
\end{abstract}

The starting thiocarbamoyl ester 2 was prepared from methyl arylacetate 1 by modified known procedure. ${ }^{10,11}$ Cyclization of thiocarbamoyl ester 2 with hydroxylamine was investigated under the various conditions (Table 1). Treatment of $\mathbf{2 a}$ with hydroxylamine hydrochloride and triethylamine (3.0 equiv) in refluxing ethanol for $17 \mathrm{~h}$ resulted in recovery of $\mathbf{2 a}$ (entry 1 ). However, 3-aminoisoxazolone 3a was produced in good yield in the presence of mercury oxide (entry 2). Cyclization with mercury (II) chloride afforded an improved yield of 3a (entry 3). We then attempted regioselective cyclization of $\mathbf{2 a}$ to 5 -aminoisoxazol-3-one 4a, based upon the literature example of the use

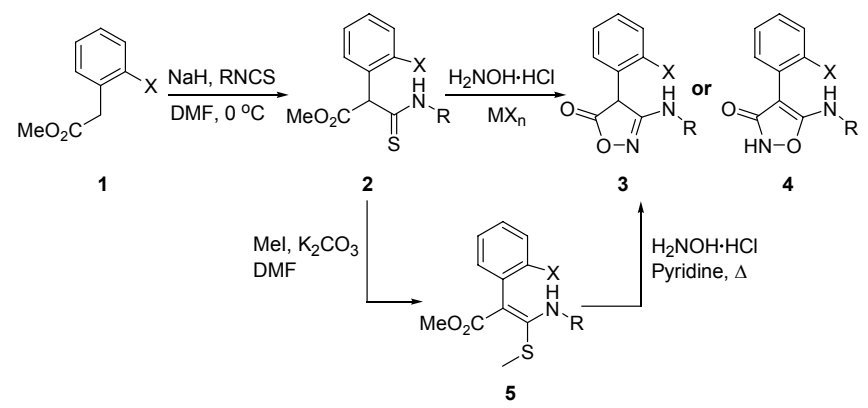

Scheme 1

Table 1. Preparation of thiocarbamoyl esters 2 and cyclization to 3-aminoisoxazol-5(4H)-ones 3 in various conditions

\begin{tabular}{|c|c|c|c|c|c|}
\hline entry & $\mathrm{X}$ & $\mathrm{R}$ & Yield of $2(\%)$ & condition & Yield of $3(\%)$ \\
\hline 1 & & & & $\mathrm{NH}_{2} \mathrm{OH} . \mathrm{HCl} / \mathrm{TEA}, \mathrm{EtOH}, 80^{\circ} \mathrm{C}$ & $3 \mathbf{a}(0)$ \\
\hline 2 & & & & $\mathrm{NH}_{2} \mathrm{OH} . \mathrm{HCl} / \mathrm{TEA}, \mathrm{HgO}, \mathrm{CH}_{2} \mathrm{Cl}_{2}, \mathrm{rt}$ & $3 a(60)$ \\
\hline 3 & & & & $\mathrm{NH}_{2} \mathrm{OH} . \mathrm{HCl} / \mathrm{TEA}, \mathrm{HgCl}_{2}, \mathrm{CH}_{2} \mathrm{Cl}_{2}, \mathrm{rt}$ & 3a (72) \\
\hline 4 & $\mathrm{Br}$ & $\mathrm{Me}$ & $\mathbf{2 a}(61)$ & $\mathrm{NH}_{2} \mathrm{OH} . \mathrm{HCl} / \mathrm{AlCl}_{3}$, toluene, $110{ }^{\circ} \mathrm{C}$ & $3 \mathbf{a}($ trace $)$ \\
\hline 5 & & & & $\mathrm{NH}_{2} \mathrm{OH} . \mathrm{HCl} / \mathrm{ZrCl}_{4}$, toluene, $110^{\circ} \mathrm{C}$ & $3 \mathbf{a}($ trace $)$ \\
\hline 7 & & & & $\mathrm{NH}_{2} \mathrm{OH} \cdot \mathrm{HCl} / \mathrm{Zr}(\mathrm{OBu}-\mathrm{t})_{4}$, toluene, $110{ }^{\circ} \mathrm{C}$ & $\mathbf{3 a}(32)$ \\
\hline 8 & & & & i. $\mathrm{MeI}, \mathrm{K} 2 \mathrm{CO} 3$, ii. $\mathrm{NH}_{2} \mathrm{OH} \cdot \mathrm{HCl} /$ pyridine, $110^{\circ} \mathrm{C}$ & $3 a(45)$ \\
\hline 9 & $\mathrm{Br}$ & $\mathrm{Ph}$ & $\mathbf{2 b}(58)$ & $\mathrm{NH}_{2} \mathrm{OH} \cdot \mathrm{HCl} / \mathrm{TEA}, \mathrm{HgCl}_{2}, \mathrm{CH}_{2} \mathrm{Cl}_{2}, \mathrm{rt}$ & $\mathbf{3 b}(52)$ \\
\hline 10 & $\mathrm{H}$ & $\mathrm{Me}$ & $2 \mathrm{c}(61)$ & $\mathrm{NH}_{2} \mathrm{OH} \cdot \mathrm{HCl} / \mathrm{TEA}, \mathrm{HgCl}_{2}, \mathrm{CH}_{2} \mathrm{Cl}_{2}, \mathrm{rt}$ & $3 \mathbf{c}(78)$ \\
\hline 11 & $\mathrm{H}$ & $\mathrm{Ph}$ & 2d (59) & $\mathrm{NH}_{2} \mathrm{OH} \cdot \mathrm{HCl} / \mathrm{TEA}, \mathrm{HgCl}_{2}, \mathrm{CH}_{2} \mathrm{Cl}_{2}, \mathrm{rt}$ & 3d (88) \\
\hline 12 & $\mathrm{Me}$ & $\mathrm{Me}$ & $2 \mathrm{e}(74)$ & $\mathrm{NH}_{2} \mathrm{OH} \cdot \mathrm{HCl} / \mathrm{TEA}, \mathrm{HgCl}_{2}, \mathrm{CH}_{2} \mathrm{Cl}_{2}, \mathrm{rt}$ & $3 e(69)$ \\
\hline 13 & OBn & $\mathrm{Me}$ & $2 f(56)$ & $\mathrm{NH}_{2} \mathrm{OH} \cdot \mathrm{HCl} / \mathrm{TEA}, \mathrm{HgCl}_{2}, \mathrm{CH}_{2} \mathrm{Cl}_{2}, \mathrm{rt}$ & $3 f(40)$ \\
\hline 14 & OBn & $\mathrm{Ph}$ & $2 \mathrm{~g}(53)$ & $\mathrm{NH}_{2} \mathrm{OH} \cdot \mathrm{HCl} / \mathrm{TEA}, \mathrm{HgCl}_{2}, \mathrm{CH}_{2} \mathrm{Cl}_{2}, \mathrm{rt}$ & $3 g(45)$ \\
\hline
\end{tabular}


of aluminum and zirconium complex in transformation of ester to amde. ${ }^{12}$ However, use of $\mathrm{AlCl}_{3}$ or $\mathrm{ZrCl}_{4}$ did not give $4 \mathbf{a}$, but afforded a trace amount of 3a instead (entry 4-6), which was presumably produced by uncatalyzed thermal cyclization reaction at elevated temperature. To examine the scope of this method in preparation of other substituted 3-aminoisoxazol-5ones, we attempted the cyclization of various thiomalonamic esters (2a-g) to the corresponding aminoisoxazoles (3a-g) as listed in Table 1. As shown in Table 1, most of the thiocarbamoyl esters were converted to the corresponding 3-aminoisoxazol5 -one under mercuric chloride mediated condition in moderate to high yield.

The structure of $\mathbf{3 a}$ was elucidated by the comparison of its spectroscopic data with those of the compound prepared via the conversion of thiocarbamoyl ester $2 \mathbf{a}$ to ketene N, S acetal 5a followed by cyclization with hydroxylamine hydrochloride in refluxing pyridine (Entry 8$).^{13}$

In summary, we have demonstrated a new and efficient method for the synthesis of 3-substituted aminoisoxazoles from readily available thiocarbamoyl esters. Mercury (II) chloride appeared to be a Lewis acid of choice among the metals tested in this cyclodesulfurization reaction. Application of this method for the synthesis of substituted aminopyrazoles is now under investigation in our laboratory.

\section{References and Notes}

1. Padmavathi, V.; Venkata Subbaiah, D. R. C.; Mahesh, K.; Lakshmi, T. R. Chem. Pharm. Bull. 2007, 55, 1704.

2. Drizin, I.; Altenbach, R. J.; Buckner, S. A.; Whiteaker, K. L.; Scott, V. E.; Darbyshire, J. F.; Jayanti, V.; Henry, R. F.; Coghlan, M. J.; Gopalakrishnan, M.; Carroll, W. A. Bioorg. Med. Chem. 2004, 12, 1895.

3. Tong, Y.; Stewart, K. D.; Thomas, S.; Przytulinska, M.; Johnson, E. F.; Klinghofer, V.; Leverson, J.; McCall, O.; Soni, N. B.; Luo, Y.; Lin, N.-h.; Sowin, T. J.; Giranda, V. L.; Penning, T. D. Bioorg. Med. Chem. Lett. 2008, 18, 5206.

4. a) Jeffery, D.; Prager, R. H.; Turner, D.; Dreimanis, M. Tetrahedron 2002, 58, 9965. b) Khalafy, J.; Setamdideh, D.; Akbari Dilmaghani, K. Molecules 2002, 7, 907.

5. Zvilichovsky, G.; Gurvich, V.; Shlomo Segev, S. J. Org. Chem. 1995, 60, 5250 .

6. a) Barbieri, W.; Bernardi, L.; Coda, S.; Palamidessi, G. Tetrahedron 1967, 23, 4395. b) Bauer, L.; Nambury, C. N. V. J. Org. Chem. 1961, 26, 4917.
7. Buyle, R.; Viehe, H. G. Tetrahedron 1969, 25, 3453.

8. Kim, C.-K.; Debellis, F.; Maggiulli, C. A. J. Heterocyclic Chem. 1987, 24, 325.

9. a) Avalos, M.; Babiano, R.; Cintas, P.; Duran, C. J.; Jimenez, J. L.; Palacios, J. C. Tetrahedron 1995, 29, 8043. b) Marchand-Brynaert, J.; Moya-Portuguez, M.; Huber, I.; Ghosez, L. J. Chem. Soc. Chem. Commun. 1983, 818. c) Foloppe, M. P.; Rault, S.; Robba, M. Tetrahedron Lett. 1992, 33, 2803. d) Stefan, V.; Kathleen, P.; Muriel, P.; Christophe, B.; Marc, C.; Andrea, V. Helv. Chim. Acta 1999, 82, 963.

10. For a related reaction, see: Krpcho, A. P.; Stephens, W. P. J. Org. Chem. 1980, 45, 1106.

11. A typical experimental procedure: To a solution of methyl 2-methylphenylacetate $(9.85 \mathrm{~g}, 59.7 \mathrm{mmol})$ in DMF $(100 \mathrm{~mL})$ was added $\mathrm{NaH}(60 \%$ in mineral oil; $2.88 \mathrm{~g}, 71.7 \mathrm{mmol})$ at $0{ }^{\circ} \mathrm{C}$. After being stirred for $30 \mathrm{~min}$, methylisothiocyanate $(4.3 \mathrm{~mL}, 62.6 \mathrm{mmol})$ was added, and the reaction mixture was stirred for $1 \mathrm{~h}$ at $\mathrm{rt}$ and poured into cold $2 \mathrm{~N} \mathrm{HCl}(100 \mathrm{~mL})$. The resulting white precipitate was collected, washed twice with water and dried in vacuo. The white solid was recrystallized from dichloromethane-hexane to give thiocarbamoyl ester $2 \mathrm{e}(10.6 \mathrm{~g}, 74 \%)$ as a white solid, $\mathrm{mp} 113-114^{\circ} \mathrm{C}$ : ${ }^{1} \mathrm{H} \mathrm{NMR}\left(200 \mathrm{MHz}, \mathrm{CDCl}_{3}\right) \delta 2.47(\mathrm{~s}, 3 \mathrm{H}), 3.19(\mathrm{~d}, J=4.9 \mathrm{~Hz}, 3 \mathrm{H})$, $5.46(\mathrm{~s}, 1 \mathrm{H}), 7.17-7.33(\mathrm{~m}, 4 \mathrm{H}), 8.72(\mathrm{bs}, 1 \mathrm{H}),{ }^{13} \mathrm{C} \mathrm{NMR}(50 \mathrm{MHz}$, $\left.\mathrm{CDCl}_{3}\right) \delta 20.0,33.2,52.8,61.2,126.2,126.7,128.5,131.4,133.4$, 137.5, 171.9, 197.9; IR (NaCl) 3315, 2951, 1733, $1539 \mathrm{~cm}^{-1}$; MS $(70 \mathrm{eV}, \mathrm{m} / z) 237\left[\mathrm{M}^{+}\right], 204,164,144,132,105$.

12. For examples of $\mathrm{Al}$ mediated activation of $\mathrm{C}=\mathrm{O}$, see; a) Basha, $\mathrm{A}$;; Lipton, M.; Weinreb, S. M. Tetrahedron Lett. 1977, 48, 4171. b) Lipton, M.; Basha, A.; Weinreb, S. M. Org. Synth.1979, 59, 49. c) Slider, D. R.; Lovelace, T. C.; MaNamara, J. M.; Reider, P. J. J. Org. Chem. 1994, 59, 1231. d) Sim, T. B.; Yoon, N. M. Synlett 1994, 827.

13. a) A typical experimental procedure for mercuric chloride mediated cyclization reactions: To a solution of thiocarbamoyl ester 2a $(0.53 \mathrm{~g}, 1.75 \mathrm{mmol})$, hydroxylamine hydrochloride $(0.13 \mathrm{~g}, 1.92$ $\mathrm{mmol})$, and mercuric chloride $(0.62 \mathrm{~g}, 2.28 \mathrm{mmol})$ in dichloromethane $(18 \mathrm{~mL})$ was added triethylamine $(0.49 \mathrm{~mL}, 3.50 \mathrm{mmol})$ at rt. The reaction mixture was stirred for $24 \mathrm{~h}$, filtered, and washed with dichloromethane $(2 \times 10 \mathrm{~mL})$. The combined organic layer was washed with $1 \mathrm{~N}$ hydrochloric acid $(20 \mathrm{~mL})$, water $(20 \mathrm{~mL})$, dried $\left(\mathrm{Na}_{2} \mathrm{SO}_{4}\right)$, and concentrated in vacuo. The remaining material was purified by flash column chromatography (hexane:ethyl acetate $=2: 1)$ to give $3 \mathbf{a}(0.34 \mathrm{~g}, 72 \%)$. b) The ${ }^{1} \mathrm{H}$ - and ${ }^{13} \mathrm{C}$ NMR spectroscopic data for compound 3a prepared from entry 3 and 8 are identical. ${ }^{1} \mathrm{H}$ NMR $\left(200 \mathrm{MHz}, \mathrm{DMSO}-d_{6}\right) \delta 2.72(\mathrm{~d}, J=5.2 \mathrm{~Hz}$, $3 \mathrm{H}), 5.00(\mathrm{~m}, 1 \mathrm{H}), 7.09-7.32(\mathrm{~m}, 3 \mathrm{H}), 7.56-7.60(\mathrm{~m}, 1 \mathrm{H}), 10.73$ (bs, 1H); ${ }^{13} \mathrm{C}$ NMR $(50 \mathrm{MHz}$, DMSO-d $) \delta 28.3,79.4,125.8$, 127.6, 129.1, 131.6, 132.6, 133.7, 164.6, 169.9; IR (KBr) 3382, $2995,1707,1616 \mathrm{~cm}^{-1} ; \mathrm{MS}(70 \mathrm{eV}, \mathrm{m} / z) 268\left[\mathrm{M}^{+}\right], 270\left[\mathrm{M}^{+}+2\right]$, 209, 195, 183, 131, 89 . 